\title{
BD-Func: A Streamlined Algorithm for Predicting Activation and Inhibition of Pathways
}

BD-Func (BiDirectional FUNCtional enrichment) is an algorithm that calculates functional enrichment by comparing lists of pre-defined genes that are known to be activated versus inhibited in a pathway or by a regulatory molecule. This paper shows that BD-Func can correctly predict cell line alternations and patient characteristics with accuracy comparable to popular algorithms, with a significantly faster run-time. BD-Func can compare scores for individual samples across multiple groups as well as provide predictive statistics and receiver operating characteristic (ROC) plots to quantify the accuracy of the signature associated with a binary phenotypic variable. BD-Func facilitates collaboration and reproducibility by encouraging users to share novel molecular signatures in the BD-Func discussion group, which is where the novel progesterone receptor and LBH589 signatures from this paper can be found. The novel LBH589 signature presented in this paper also serves as a case study showing how a custom signature using cell line data can accurately predict activity in vivo. This software is available to download at https://sourceforge.net/projects/bdfunc/. 
5 Bioinformatics Core, Department of Molecular Medicine, ${ }^{2}$ Department of Cancer Biology, City of Hope

6 National Medical Center, Duarte, CA 91010

7 * To whom correspondence should be addressed. Tel: 626-256-4673; Fax: 626-471-3708; Email: cwarden@coh.org or 8 yyuan@coh.org

\section{ABSTRACT} molecule. This paper shows that BD-Func can correctly predict cell line alternations and patient characteristics with

13 accuracy comparable to popular algorithms, with a significantly faster run-time. BD-Func can compare scores for

14 individual samples across multiple groups as well as provide predictive statistics and receiver operating characteristic

15 (ROC) plots to quantify the accuracy of the signature associated with a binary phenotypic variable. BD-Func

16 facilitates collaboration and reproducibility by encouraging users to share novel molecular signatures in the BD-Func

17 discussion group, which is where the novel progesterone receptor and LBH589 signatures from this paper can be

18 found. The novel LBH589 signature presented in this paper also serves as a case study showing how a custom

19 signature using cell line data can accurately predict activity in vivo. This software is available to download at https://sourceforge.net/projects/bdfunc/. 
Systems-level analysis of the combined expression pattern of multiple genes can be more informative than the expression pattern of an individual gene, and there are a number of tools to calculate functional enrichment of differentially expressed genes (Huang et al. 2009; Naeem et al. 2012; Nam \& Kim 2008). However, many functional annotations merely list membership in a pathway or ontology without explicitly modelling genes that should show activation or inhibition. For example, consider the KEGG canonical Wnt signalling pathway (Figure 1) (Kanehisa \& Goto 2000). This gene list includes molecules that both activate and inhibit the pathway, resulting in different phenotypes (Dellinger et al. 2012; Logan \& Nusse 2004). However, many functional enrichment tools would expect all the members of the pathway to behave similarly (Figure 1C), such that up-regulation of a mix of activators and inhibitors can receive a higher score than selective up-regulation of only activators within the pathway. For example, the most standard method for functional enrichment is to calculate over-representation of one gene list in another gene list, possibly using a Fisher's exact test or hypergeometric test; in the example described above, this sort of statistical test would ask if a list of differentially expressed genes shows a higher number of Wnt signalling genes than expected by chance. This sort of test cannot differentiate the behavior of those genes unless more detailed gene lists are defined (such as Wnt-inhibitors versus Wnt-agonists). This is a basic problem that BD-Func (BiDirectional FUNCtional enrichment) is designed to overcome.

Most functional enrichment tools either require upstream filtering of gene lists (FuncAssociate (Berriz et al. 2009), GATHER (Chang \& Nevins 2006), DAVID (Huang et al. 2008), Connectivity Map (Lamb et al. 2006), WebGestalt (Zhang et al. 2005), GoMiner (Zeeberg et al. 2003), ErmineJ (Lee et al.

41 2005)) and/or a comparison of signal intensities between two groups (T-profiler (Boorsma et al. 2005), GSVA (ㅂanzelmann et al. 2013), PAGE (Kim \& Volsky 2005), GSEA (ubramanian et al. 2005), ErmineJ (Lee et al. 2005)). However, BD-Func compares the relative expression levels between activated and inhibited genes, and we show that BD-Func can successfully analyze either fold-change values between populations or raw intensity / expression values (for both microarray and RNA-Seq data). Signalling Pathway Impact Analysis (Draghici et al. 2007; Tarca et al. 2008) can be used to model activation and

47 inhibition within a graph, but that algorithm primarily focuses on network topology (which is not always known); in contrast, BD-Func uses a simpler assumption of binning genes into two categories (activation 
feature only present in a limited number of functional genomic tools. For example, ASSESS (Edelman et al. 2006) can theoretically predict functional enrichment in a single sample, but that score is not taken into context amongst other samples: in other words, BD-Func uniquely uses a single sample enrichment score as a classifier, which may be useful for personalized medicine research or for the development of novel molecular signatures where the user may need to quantify the utility of the signature as a classifier. Additionally, correlations between a single sample and various samples within a database (such as SPIED (Williams 2012)) can provide information about a single sample, but this requires having a database of samples for comparison (so, this strategy will not work without the presence of an external database).

Finally, BD-Func users are encouraged to share their lists of activated and inhibited genes in a simple file format. This allows easy application of models that may not be in an existing database for molecular signatures.

This study tests the accuracy of BD-Func on datasets that were used to define gene sets in MSigDB (Molecular Signatures DataBase, (Subramanian et al. 2005)), in comparison to GSEA (Gene Set Enrichment Analysis, (Mootha et al. 2003; Subramanian et al. 2005)) and IPA (Ingenuity Pathway Analysis, Ingenuity ${ }^{\circledR}$ Systems, www.ingenuity.com). IPA was selected for comparison because the upstream regulator function utilizes a similar principle as BD-Func (activation and inhibition is predicted by comparing the proportion of activated or inhibited targets, based upon annotations in the proprietary IPA database). GSEA was selected for comparison for two reasons: 1) GSEA was specifically designed to analyze MSigDB signatures, thus serving as a good baseline for positive control datasets and 2) MSigDB contains some signatures for both up- and down-regulated genes, so it is useful to compare separate analysis of these signatures (using GSEA) versus a direct comparison of up-regulated to down-regulated

71 gene expression (using BD-Func). Different models for TGF $\beta$ and progesterone receptor (PGR) activity

72 are also tested for robustness by application to other datasets. Finally, the utility of BD-Func to study custom gene signatures is tested with a novel signature associated with progesterone receptor status in breast cancer patients as well as a novel LBH589 signature that was defined using previously published cell line data and is validated using novel in vivo data presented in this study. 
The basic principle behind BD-Func is to treat activated and inhibited genes as replicate observations in two populations. BD-Func is agnostic towards the type of signal used for analysis. For example, this paper uses BD-Func to study both fold-change values between two populations as well as raw signal intensities for a single column of signal values. In the paper, the t-test statistic is used to compare the expression patterns of activated and inhibited genes. However, BD-Func also allows users to compare the activated and inhibited distributions using a Mann-Whitney $U$ test or Kolmogorov-Smirnov (K-S) test. BD-Func users have the option to calculate False Discovery Rates (FDR) using the method of Benjamini and Hochberg (Benjamini \& Hochberg 1995) or the Storey q-value (

BD-Func comes with four enrichment files: c2, c5, and c6 from MSigDB (Liberzon et al. 2011) and a list of functions defined directly from the human Gene Ontology (GO) annotations (Ashburner et al. 2000). All of these lists were created by searching for functions with both "up" and "down" (or "positive regulation" and "negative regulation") gene lists. For the human GO file, a functional annotation needed to contain at least 10 positively regulated genes and 10 negatively regulated genes. We also encourage users to share their own custom models on the BD-Func discussion group: http://sourceforge.net/p/bdfunc/discussion/

There are three different input files that can be analyzed using BD-Func (Figure S1):

1-D Input File: If the user supplies expression values for a single column of data, a density plot is created for the signal for the activated and inhibited genes. In this case, the BD-Func algorithm works exactly as described above.

2-D Input File: If the input file contains multiple columns of data, BD-Func is first run separately for each sample (represented by a column in the data matrix), as described above. Next, box-plots are created for test-statistic scores for each group (labelled in the header of the input file; Figure S2). Finally, an ANOVA p-value is provided to compare the test statistics between groups. Theoretically, test statistics could be used to make functional predictions for each sample in isolation. There are some examples in this paper where this strategy works OK. However, comparing test statistics across all samples within different groups is the only strategy that consistently works well for all the analysis presented in this paper. 
104

105 "positive" and "negative"), BD-Func will create a receiver operating characteristic (ROC) plot using the

2-D Input File for Classifier: If the input file contains multiple columns of data and two groups (called

test-statistic as the classification score using the ROCR package (Sing et al. 2005). This is in addition to all the calculations and output files for a normal 2-D input file containing multiple columns for BD-Func to analyze (in this paper, this represents normalized signal intensity values).

\section{Sample Acquisition and Processing}

Microarray datasets were downloaded from GEO (Edgar et al. 2002) or Array-Express (Parkinson et al. 2009). When raw .CEL files were available, samples were RMA normalized (Irizarry et al. 2003). Otherwise, processed intensity values were used for microarray analysis. Fold-Change values for all of the cell line datasets (TGF $\beta$ (Padua et al. 2008; Qin et al. 2009; Renzoni et al. 2004; Sartor et al. 2010;

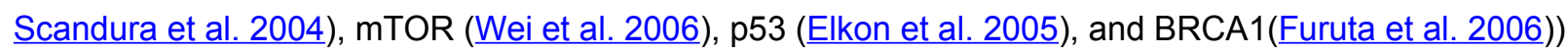
and the MSigDB progesterone receptor dataset (Claus et al. 2008) were calculated using the method of the least-squares mean using Partek ${ }^{\circledR}$ Genomics Suite ${ }^{\mathrm{TM}}$ (Partek Inc. 2012).

All other clinical samples (Anders et al. 2008; Bild et al. 2006; Chin et al. 2006; Finak et al. 2008; Huang et al. 2003; Ivshina et al. 2006; Sotiriou et al. 2003; The Cancer Genome Atlas Network 2012) were downloaded and analyzed for differential expression using BRAVO (http://bravo.coh.org/) (Deng et al. 2013, unpublished data). The novel progesterone receptor gene signature presented in this paper was produced by identifying genes in the expO dataset (GEO Series GSE2109) with a |fold-change| > 2 and an False Discovery Rate (FDR) $<0.05$ (where the FDR is calculated using the method of Benjamini and Hochberg (Benjamini \& Hochberg 1995) to analyze the distribution of t-test p-values). This is how the samples in this particular paper were processed, but users are not required to use this particular set of tools for preparing BD-Func input files and/or creating gene lists for custom signatures.

Reads Per Kilobase per Million mapped reads (RPKM (Mortazavi et al. 2008)) values for RNASeq data was downloaded from the TCGA web portal (The Cancer Genome Atlas Network 2012). RPKM values were transformed by addition of 0.1 (to avoid large fold-change values for low coverage reads) followed by $a \log _{2}$ transformation (to normalize the signal distribution).

\section{GSEA Comparison}



version 3.1) for GSEA analysis: TGFB_UP.V1 (Padua et al. 2008), MTOR_UP.N4.V1 (Wei et al. 2006), P53_DN.V2 (Elkon et al. 2005), and BRCA_DN.V1 (Furuta et al. 2006). GSEA (( ,version 2.0) calculated p-values by permutation over phenotypes whenever possible (Anders et al. 2008; Chin et al. 2006; Claus et al. 2008; Elkon et al. 2005; Finak et al. 2008; Huang et al. 2003; Padua et al. 2008; Sartor et al. 2010; Scandura et al. 2004; The Cancer Genome Atlas Network 2012; Wei et al. 2006), although there were a few datasets with less than 3 replicates for which gene sets had to be permuted instead of phenotypes (Furuta et al. 2006; Qin et al. 2009; Renzoni et al. 2004). For recovery of known perturbations of oncogenic regulators, GSEA results must either show a FWER p-value $<0.25$ or a NOM p-value $<0.05$, which are the default cut-offs

$\underline{\text { IPA Comparison }}$

Ingenuity Pathway Analysis (IPA, Ingenuity® Systems, www.ingenuity.com) contains an "Upstream

Regulator" module that compares the enrichment of activated and inhibited genes among up- and downregulated genes. So, the underlying principle is similar to BD-Func except it utilizes Ingenuity's propriety database of regulatory interactions and uses a z-score to calculate statistical significance between activated and inhibited genes. Gene lists in IPA were filtered for those genes showing |fold-change| $>1.5$ while the entire gene list is used to define background enrichment. For recovery of known perturbations of oncogenic regulators, the upstream regulator must be identified as "activated" or "inhibited" (|z-score| > 2),

151 which are the default cut-offs

\section{$\underline{\text { LBH589 Signature }}$}

Activated and inhibited genes were defined using overlapping gene lists defined from 3 cell line treatments that have been previously published (Kubo et al. 2013). That same study showed that LBH589

155 treatment significantly decreased tumor volume in exemestane (EXE) resistant MCF-7aro xenografts in 156 mice. This study analyzes novel microarray data from EXE-resistant tumors treated with (EXE + LBH589) 
158

159

160

161

162

163

164

165

166

167

168

169

170

171

172

173

174

175

176

177

178

179

180

181

182

183

184

Hope Institutional Animal Care and Use Committee. This novel microarray data is available in GEO series GSE47346.

In order to be included in the novel BD-Func signature, genes must show differential expression in all 3 cell lines. Genes were defined as differentially expressed if they showed a $\mid$ fold-change $\mid>1.5$ and $p$ value $<0.05$, and the BD-Func signature genes had to meet these conditions for each of the 3 LBH589 cell line treatments (with consistent direction of fold-change). P-values were calculated via 1-way ANOVA with appropriate linear contrast was used to compare data sets using Partek ${ }^{\circledR}$ Genomics Suite $^{\mathrm{TM}}$ (Partek, Inc., St. Louis, MO). Fold-change values were calculated based upon the least-squares mean, and data was normalized using robust multichip average (RMA) normalization (Irizarry et al. 2003).

\section{RESULTS}

\section{BD-Func Shows Equal or Greater Performance to GSEA and IPA for Functional Enrichment}

Given the relative ease by which samples can be classified as having positive or negative activity for an individual biomarker, the accuracy of BD-Func was first tested by applying several MSigDB oncogenic signatures (Liberzon et al. 2011) to the datasets from which the signatures were defined (Claus et al. 2008; Elkon et al. 2005; Furuta et al. 2006; Padua et al. 2008; Wei et al. 2006). BD-Func was able to detect the activation or inhibition of all of these oncogenic signatures (Table 1, Figure 2). GSEA could detect all of the signatures except the Claus et al. (Claus et al. 2008) progesterone receptor signature. Among these 5 test datasets, IPA could only detect the activity of 2 of these genes; however, this is not a completely fair comparison because we would expect some over fitting of the MSigDB signatures for the GSEA and BD-Func analysis. Nevertheless, the significance of this analysis is that BD-Func can accurately detect perturbation of all of these biomarkers on datasets where we know that these specific genes will be altered.

In order to test the performance of BD-Func, GSEA, and IPA on novel datasets (which were not used by MSigDB to define gene lists), we applied the 3 algorithms to four datasets with TGF $\beta$ treatments (Table 2). All 3 algorithms showed roughly equal performance for predicting TGF $\beta$ treatment in the appropriate samples. Overall, analyses of these nine datasets indicate that BD-Func can provide similar quality results as GSEA and IPA. 


\section{BD-Func Can Determine the Accuracy of Novel Predictive Models}

Six breast cancer datasets were also used to test the robustness of the progesterone receptor


Genome Atlas Network 2012)). Unfortunately, neither BD-Func, GSEA, nor IPA could predict progesterone status in all seven patient populations (Table 3). To be fair, the original Claus et al. (Claus et al. 2008) dataset was used to define progesterone receptor status in meningioma samples whereas the novel datasets tested were all breast cancer samples (where testing for over-expression of progesterone receptor is common (Bardou et al. 2003)). Nevertheless, BD-Func is designed to be able to utilize custom gene signatures with activated and inhibited, so we defined a novel progesterone receptor signature using the expO dataset (GEO Series GSE2109). This signature can identify progesterone receptor positive and negative patients for all 7 cohorts ( 1 meningioma and 6 breast cancer datasets), so it is robust enough for application to multiple cancer types.

Another unique feature of BD-Func is the ability to use the activation versus inhibition test statistic as a classifier to define a predictive model. If a t-test statistic of 2 is used for the cut-off of distinguishing the positive and negative populations (roughly corresponding to a p-value $<0.05$ ), it is clear that the MSigDB signature is extremely accurate at predicting PGR status in the original dataset but not in the breast cancer datasets (Table S1). Likewise, the TGF $\beta$ signature could differentiate between the treated and untreated groups if the test statistic of 2 was used as the threshold to distinguish the groups (Figure S2). However, this threshold does not work well in all circumstances: unlike the analysis of fold-change values, the p-value (for any statistical method) is not always the ideal statistic for assessment of functional enrichment on intensity values. For example, the mTOR and BRCA1 signatures (Figure 2B) show appropriate changes in test statistics that clearly distinguish treated and untreated groups, but activation and inhibition can't be defined based upon a pre-defined cut-off for the test statistic value (e.g. 2 or -2). For this reason, we provide an ANOVA p-value to quantify the difference in test-statistic between groups, where the test statistic serves as a score for a second calculation of statistical significance.

Additionally, we believe that predictive statistics are a useful method for accessing BD-Func scores for individual samples within large patient populations. In order to quantify the accuracy of the model without depending on a pre-defined cut-off, BD-Func produces receiver operating characteristic 
$213(R O C)$ plots for each cohort and the area under the curve (AUC) is calculated for each ROC plot (where a

$214100 \%$ accurate model would have an $A U C=1.00$ ) (Figure S3, Table S1). The superior performance of the

215 novel PGR signature on the breast cancer cohorts becomes even clearer when these predictive statistics

216 are compared for the two models (Figure 3, Figure S4, and Table S2). Importantly, the novel signature

217 showed the same level of accuracy on the TCGA breast cancer dataset as the original expO dataset. This

218 is significant because the TCGA dataset is over twice as large as the expO dataset, and the TCGA dataset

219 utilizes RNA-Seq while the expO dataset utilizes microarrays to quantify gene expression. In other words,

220 this shows that BD-Func is capable of producing very robust predictions that translate across different

221 genomic technologies.

222

223

224

225

226

227

228

229

230

231

232

233

\section{BD-Func Accurately Quantifies LBH589 Activity Using A Novel Signature}

LBH589 (panobinostat) is a histone deacetylase inhibitor that has been previously shown to suppress the proliferation of aromatase inhibitor resistant breast cancer cells, which was a conclusion supported in part by functional enrichment analysis of commonly affected genes from 3 cell line experiments (Kubo et al. 2013). Gene lists derived from these cell line experiments can be easily used to define a BD-func signature, so we hypothesized that the results from this previous in vitro cell culture study could be used to predict LBH589 activity in vivo in an animal study. Specifically, we asked if the signature defined based upon LBH589 treatment in 3 cell lines (H295R, MCF-7her2, HeLa) could detect LBH589 activity in a mouse xenograft from a different cell line (MCF-7aro xenograft treated with EXE). Indeed, BD-Func correctly used the cell line LBH589 signature to identify common gene expression changes in the tumours treated with LBH589 and EXE compared to the mice that were only exposed to EXE treatment (Figure 4).

\section{DISCUSSION}

Comparison of BD-Func to GSEA indicated that BD-Func can provide similar oncogenic signature predictions with much shorter run time (Table S3) and a more direct comparison of genes that are expected to be up- or down-regulated by the oncogenic regulators. One limitation to BD-Func is that it can only conduct functional enrichment for regulators with genes that are both up- and down-regulated, so there are many gene lists in MSigDB that cannot be analyzed using BD-Func (which instead should be 
analyzed using a tool such as GSEA). It is also worth noting that BD-Func can work with a wide range of

241 sizes of gene lists for activated and inhibited genes (Table S4), but we would recommend using at least a

242 few dozen genes when defining custom signatures.

that both programs could provide similar performance, which is not surprising giving the design of that module in IPA. One benefit to utilizing IPA is that IPA has a curated database which lists with a wider variety of regulators than the MSigDB oncogenic signatures that can be analyzed in BD-Func. In contrast, one major benefit to using BD-Func is the greater theoretical range of applications. For example, BD-Func provides an enrichment file for Gene Ontology (GO (Ashburner et al. 2000)) categories, but IPA does utilize this same strategy of analyzing functional ontologies by comparing the expression of positively and negatively regulated genes.

The Connectivity Map is a commonly used tool to study gene expression profiles for drugs and other chemical perturbations (Lamb et al. 2006). There are no LBH589 / panobinostat treatments in the Connectivity Map database (although this database can certainly provide other useful information), so BDFunc provides a unique opportunity to test for gene signatures that show a strong positive or negative correlation with novel drug treatments (such as LBH589). Additionally, BD-Func is compatible with any gene mapping (in this case, gene symbol), whereas the Connectivity Map requires users to define their signatures in terms of HG-U133A probes. For example, affected gene symbols had to be converted to HG-U133A probes for this analysis. We believe that being able to define signatures based upon gene symbol is a substantial practical benefit.

BD-Func also calculates a test statistic to represent functional activation or inhibition for each individual sample in a dataset, and this study shows how this statistic can be directly used as a classifier that can be used to quantify the predictive power of a given functional model. More specifically, this study shows the utility of using BD-Func for applying two novel predictive models (for progesterone receptor status in patients and for LBH589 drug treatment). The LBH589 signature provided biological confirmation

265 that the results from an in vitro model can indeed apply to validation experiments in vivo. This is important 266 because our hope is that the streamlined analysis, simple input file design, and BD-Func discussion board 267 can be used to help scientists quickly and easily share novel predictive models. In short, BD-Func 
provides a novel framework for functional enrichment (by comparing the relative expression of activated

269 versus inhibited genes) that is freely available with a user interface that is accessible to biologists without

270 any coding experience. The results of this paper show that BD-Func provides accurate predictions

271 matched by other popular tools, which make it a useful complement to standard analysis using tools like

272 GSEA or IPA.

\section{ACKNOWLEDGEMENTS}

274 We would like to thank Christine Brown, Mike Barish, and Thanh Dellinger for discussions that led to the

275 creation of this algorithm. We would like to thank Xiwei Wu, Zheng Liu, and two anonymous reviewers for

276 discussions regarding the BD-Func algorithm. We would like to thank the City of Hope Functional

277 Genomics Core for processing the microarray data. 
Anders CK, Acharya CR, Hsu DS, Broadwater G, Garman K, Foekens JA, Zhang Y, Wang Y, Marcom K, Marks JR, Mukherjee S, Nevins JR, Blackwell KL, and Potti A. 2008. Age-Specific Differences in Oncogenic Pathway Deregulation Seen in Human Breast Tumors. PLoS ONE 3:e1373.

Ashburner M, Ball CA, Blake JA, Botstein D, Butler H, Cherry JM, Davis AP, Dolinski K, Dwight SS, Eppig JT, Harris MA, Hill DP, Issel-Tarver L, Kasarskis A, Lewis S, Matese JC, Richardson JE, Ringwald M, Rubin GM, and Sherlock G. 2000. Gene Ontology: tool for the unification of biology. Nat Genet 25:25-29.

Bardou V-J, Arpino G, Elledge RM, Osborne CK, and Clark GM. 2003. Progesterone Receptor Status Significantly Improves Outcome Prediction Over Estrogen Receptor Status Alone for Adjuvant Endocrine Therapy in Two Large Breast Cancer Databases. Journal of Clinical Oncology 21:19731979.

Benjamini Y, and Hochberg Y. 1995. Controlling the False Discovery Rate: A Practical and Powerful Approach to Multiple Testing. Journal of the Royal Statistical Society Series B (Methodological) 57:289-300.

Berriz GF, Beaver JE, Cenik C, Tasan M, and Roth FP. 2009. Next generation software for functional trend analysis. Bioinformatics 25:3043-3044.

Bild AH, Yao G, Chang JT, Wang Q, Potti A, Chasse D, Joshi M-B, Harpole D, Lancaster JM, Berchuck A, Olson JA, Marks JR, Dressman HK, West M, and Nevins JR. 2006. Oncogenic pathway signatures in human cancers as a guide to targeted therapies. Nature 439:353-357.

Boorsma A, Foat BC, Vis D, Klis F, and Bussemaker HJ. 2005. T-profiler: scoring the activity of predefined groups of genes using gene expression data. Nucleic Acids Research 33:W592-W595.

Chang JT, and Nevins JR. 2006. GATHER: a systems approach to interpreting genomic signatures. Bioinformatics 22:2926-2933.

Chin K, DeVries S, Fridlyand J, Spellman PT, Roydasgupta R, Kuo W-L, Lapuk A, Neve RM, Qian Z, Ryder T, Chen F, Feiler H, Tokuyasu T, Kingsley C, Dairkee S, Meng Z, Chew K, Pinkel D, Jain A, Ljung BM, Esserman L, Albertson DG, Waldman FM, and Gray JW. 2006. Genomic and transcriptional aberrations linked to breast cancer pathophysiologies. Cancer Cell 10:529-541.

Claus EB, Park PJ, Carroll R, Chan J, and Black PM. 2008. Specific Genes Expressed in Association with Progesterone Receptors in Meningioma. Cancer Research 68:314-322.

Dellinger TH, Planutis K, Jandial DD, Eskander RN, Martinez ME, Zi X, Monk BJ, and Holcombe RF. 2012. Expression of the Wnt antagonist Dickkopf-3 is associated with prognostic clinicopathologic characteristics and impairs proliferation and invasion in endometrial cancer. Gynecologic Oncology 126:259-267.

Deng X, Warden C, Liu Z, Zhang I, and Yuan Y-C. 2013, unpublished data. BRAVO: biomarkers recognition and validation online. in preparation.

Draghici S, Khatri P, Tarca AL, Amin K, Done A, Voichita C, Georgescu C, and Romero R. 2007. A systems biology approach for pathway level analysis. Genome Research 17:000.

Edelman E, Porrello A, Guinney J, Balakumaran B, Bild A, Febbo PG, and Mukherjee S. 2006. Analysis of sample set enrichment scores: assaying the enrichment of sets of genes for individual samples in genome-wide expression profiles. Bioinformatics 22:e108-e116.

Edgar R, Domrachev M, and Lash AE. 2002. Gene Expression Omnibus: NCBI gene expression and hybridization array data repository. Nucleic Acids Research 30:207-210.

Elkon R, Rashi-Elkeles S, Lerenthal Y, Linhart C, Tenne T, Amariglio N, Rechavi G, Shamir R, and Shiloh Y. 2005. Dissection of a DNA-damage-induced transcriptional network using a combination of microarrays, RNA interference and computational promoter analysis. Genome Biology 6:R43.

Finak G, Bertos N, Pepin F, Sadekova S, Souleimanova M, Zhao H, Chen H, Omeroglu G, Meterissian S, Omeroglu A, Hallett M, and Park M. 2008. Stromal gene expression predicts clinical outcome in breast cancer. Nat Med 14:518-527.

Furuta S, Wang J-M, Wei S, Jeng Y-M, Jiang X, Gu B, Chen P-L, Lee EYHP, and Lee W-H. 2006. Removal of BRCA1/CtIP/ZBRK1 repressor complex on ANG1 promoter leads to accelerated mammary tumor growth contributed by prominent vasculature. Cancer Cell 10:13-24.

Hanzelmann S, Castelo R, and Guinney J. 2013. GSVA: gene set variation analysis for microarray and RNA-Seq data. BMC Bioinformatics 14:7.

Huang DW, Sherman BT, and Lempicki RA. 2008. Systematic and integrative analysis of large gene lists using DAVID bioinformatics resources. Nat Protocols 4:44-57. 
Huang DW, Sherman BT, and Lempicki RA. 2009. Bioinformatics enrichment tools: paths toward the comprehensive functional analysis of large gene lists. Nucleic Acids Research 37:1-13.

Huang E, Cheng SH, Dressman H, Pittman J, Tsou MH, Horng CF, Bild A, Iversen ES, Liao M, Chen CM, West M, Nevins JR, and Huang AT. 2003. Gene expression predictors of breast cancer outcomes. The Lancet 361:1590-1596.

Irizarry RA, Hobbs B, Collin F, Beazerâ€ $\square$ Barclay YD, Antonellis KJ, Scherf U, and Speed TP. 2003. Exploration, normalization, and summaries of high density oligonucleotide array probe level data. Biostatistics 4:249-264.

Ivshina AV, George J, Senko O, Mow B, Putti TC, Smeds J, Lindahl T, Pawitan Y, Hall P, Nordgren H, Wong JEL, Liu ET, Bergh J, Kuznetsov VA, and Miller LD. 2006. Genetic Reclassification of Histologic Grade Delineates New Clinical Subtypes of Breast Cancer. Cancer Research 66:10292-10301.

Kanehisa M, and Goto S. 2000. KEGG: Kyoto Encyclopedia of Genes and Genomes. Nucleic Acids Research 28:27-30.

Kim S-Y, and Volsky D. 2005. PAGE: Parametric Analysis of Gene Set Enrichment. BMC Bioinformatics 6:144.

Kubo M, Kanaya N, Petrossian K, Ye J, Warden C, Liu Z, Nishimura R, Osako T, Okido M, Shimada K, Takahashi M, Chu P, Yuan Y-C, and Chen S. 2013. Inhibition of the proliferation of acquired aromatase inhibitor-resistant breast cancer cells by histone deacetylase inhibitor LBH589 (panobinostat). Breast Cancer Research and Treatment 137:93-107.

Lamb J, Crawford ED, Peck D, Modell JW, Blat IC, Wrobel MJ, Lerner J, Brunet J-P, Subramanian A, Ross KN, Reich M, Hieronymus H, Wei G, Armstrong SA, Haggarty SJ, Clemons PA, Wei R, Carr SA, Lander ES, and Golub TR. 2006. The Connectivity Map: Using Gene-Expression Signatures to Connect Small Molecules, Genes, and Disease. Science 313:1929-1935.

Lee H, Braynen W, Keshav K, and Pavlidis P. 2005. ErmineJ: Tool for functional analysis of gene expression data sets. BMC Bioinformatics 6:269.

Liberzon A, Subramanian A, Pinchback R, Thorvaldsdóttir H, Tamayo P, and Mesirov JP. 2011. Molecular signatures database (MSigDB) 3.0. Bioinformatics 27:1739-1740.

Logan CY, and Nusse R. 2004. THE WNT SIGNALING PATHWAY IN DEVELOPMENT AND DISEASE. Annual Review of Cell and Developmental Biology 20:781-810.

Mootha VK, Lindgren CM, Eriksson K-F, Subramanian A, Sihag S, Lehar J, Puigserver P, Carlsson E, Ridderstrale M, Laurila E, Houstis N, Daly MJ, Patterson N, Mesirov JP, Golub TR, Tamayo P, Spiegelman B, Lander ES, Hirschhorn JN, Altshuler D, and Groop LC. 2003. PGC-1[alpha]responsive genes involved in oxidative phosphorylation are coordinately downregulated in human diabetes. Nat Genet 34:267-273.

Mortazavi A, Williams BA, McCue K, Schaeffer L, and Wold B. 2008. Mapping and quantifying mammalian transcriptomes by RNA-Seq. Nat Meth 5:621-628.

Naeem H, Zimmer R, Tavakkolkhah P, and Küffner R. 2012. Rigorous assessment of gene set enrichment tests. Bioinformatics 28:1480-1486.

Nam D, and Kim S-Y. 2008. Gene-set approach for expression pattern analysis. Briefings in Bioinformatics 9:189-197.

Padua D, Zhang XHF, Wang Q, Nadal C, Gerald WL, Gomis RR, and Massagué J. 2008. TGF $\beta$ Primes Breast Tumors for Lung Metastasis Seeding through Angiopoietin-like 4. Cell 133:66-77.

Parkinson H, Kapushesky M, Kolesnikov N, Rustici G, Shojatalab M, Abeygunawardena N, Berube H, Dylag M, Emam I, Farne A, Holloway E, Lukk M, Malone J, Mani R, Pilicheva E, Rayner TF, Rezwan F, Sharma A, Williams E, Bradley XZ, Adamusiak T, Brandizi M, Burdett T, Coulson R, Krestyaninova M, Kurnosov P, Maguire E, Neogi SG, Rocca-Serra P, Sansone S-A, Sklyar N, Zhao M, Sarkans U, and Brazma A. 2009. ArrayExpress update-from an archive of functional genomics experiments to the atlas of gene expression. Nucleic Acids Research 37:D868-D872. Partek Inc. 2012. Partek $®$ Genomics Suite, Version 6.6. Version 6.6, revision 6.12.1011 ed. St. Louis. Qin H, Chan M, Liyanarachchi S, Balch C, Potter D, Souriraj I, Cheng A, Agosto-Perez F, Nikonova E, Yan P, Lin H-J, Nephew K, Saltz J, Showe L, Huang T, and Davuluri R. 2009. An integrative ChIP-chip and gene expression profiling to model SMAD regulatory modules. BMC Systems Biology 3:73.

Renzoni E, Abraham D, Howat S, Shi-Wen X, Sestini P, Bou-Gharios G, Wells A, Veeraraghavan S, Nicholson A, Denton C, Leask A, Pearson J, Black C, Welsh K, and du Bois R. 2004. Gene expression profiling reveals novel TGFbeta targets in adult lung fibroblasts. Respiratory Research 5:24. 
Sartor MA, Mahavisno V, Keshamouni VG, Cavalcoli J, Wright Z, Karnovsky A, Kuick R, Jagadish HV, Mirel B, Weymouth T, Athey B, and Omenn GS. 2010. ConceptGen: a gene set enrichment and gene set relation mapping tool. Bioinformatics 26:456-463.

Scandura JM, Boccuni P, Massagué J, and Nimer SD. 2004. Transforming growth factor $\beta$-induced cell cycle arrest of human hematopoietic cells requires p57KIP2 up-regulation. Proceedings of the National Academy of Sciences of the United States of America 101:15231-15236.

Sing T, Sander O, Beerenwinkel N, and Lengauer T. 2005. ROCR: visualizing classifier performance in R. Bioinformatics 21:3940-3941.

Sotiriou C, Neo S-Y, McShane LM, Korn EL, Long PM, Jazaeri A, Martiat P, Fox SB, Harris AL, and Liu ET. 2003. Breast cancer classification and prognosis based on gene expression profiles from a population-based study. Proceedings of the National Academy of Sciences 100:10393-10398.

Storey JD, and Tibshirani R. 2003. Statistical significance for genomewide studies. Proceedings of the National Academy of Sciences 100:9440-9445.

Subramanian A, Tamayo P, Mootha VK, Mukherjee S, Ebert BL, Gillette MA, Paulovich A, Pomeroy SL, Golub TR, Lander ES, and Mesirov JP. 2005. Gene set enrichment analysis: A knowledge-based approach for interpreting genome-wide expression profiles. Proceedings of the National Academy of Sciences of the United States of America 102:15545-15550.

Tarca AL, Draghici S, Khatri P, Hassan SS, Mittal P, Kim J-S, Kim CJ, Kusanovic JP, and Romero R. 2008. A Novel Signaling Pathway Impact Analysis (SPIA). Bioinformatics.

The Cancer Genome Atlas Network. 2012. Comprehensive molecular portraits of human breast tumours. Nature 490:61-70.

Wei G, Twomey D, Lamb J, Schlis K, Agarwal J, Stam RW, Opferman JT, Sallan SE, den Boer ML, Pieters R, Golub TR, and Armstrong SA. 2006. Gene expression-based chemical genomics identifies rapamycin as a modulator of MCL1 and glucocorticoid resistance. Cancer Cell 10:331-342.

Williams G. 2012. A searchable cross-platform gene expression database reveals connections between drug treatments and disease. BMC Genomics 13:12.

Zeeberg B, Feng W, Wang G, Wang M, Fojo A, Sunshine M, Narasimhan S, Kane D, Reinhold W, Lababidi S, Bussey K, Riss J, Barrett J, and Weinstein J. 2003. GoMiner: a resource for biological interpretation of genomic and proteomic data. Genome Biology 4:R28.

Zhang B, Kirov S, and Snoddy J. 2005. WebGestalt: an integrated system for exploring gene sets in various biological contexts. Nucleic Acids Research 33:W741-W748. 


\section{Figure 1}

Many Pathways are Characterized by a Mix of Activation and Inhibition

This figure shows the initial signalling steps in the Wnt signalling pathway, as defined by KEGG ( Kanehisa \& Goto 2000 ). Up-regulated genes are shown in red and down-regulated genes are shown in green. A. Complete Activation All genes activating the Wnt signalling pathway are up-regulated and all inhibitors are down-regulated. B. Complete Inhibition All genes activating the Wnt signalling pathway are down-regulated and all inhibitors are up-regulated. C. Mixed Pattern All genes in the figure are up-regulated. It is unclear what the downstream expression levels should be, but one may hypothesize a mixed result from Figure 1A and Figure 1B. However, most functional enrichment tools would predict this as the pattern with the strongest up-regulation. Underneath each diagram is the expected signal distribution that would be produced by BD-Func.

\section{Figure 1: Many Pathways are Characterized by a Mix of Activation and Inhibition}

A. Complete Activation
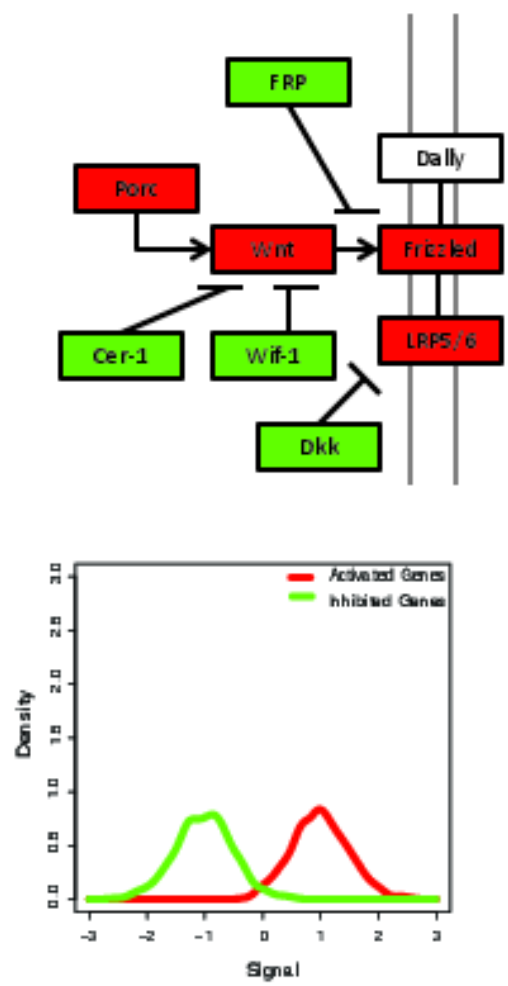

B. Complete Inhibition
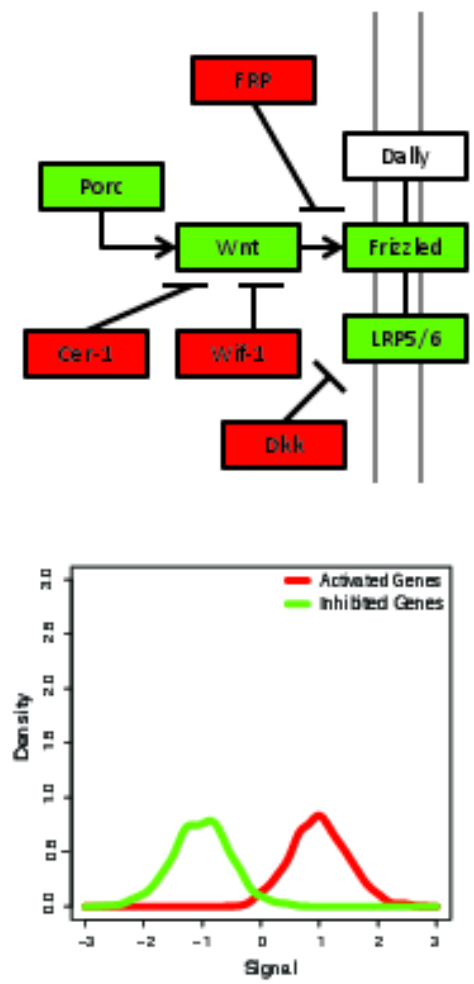

C. Mixed Pattern
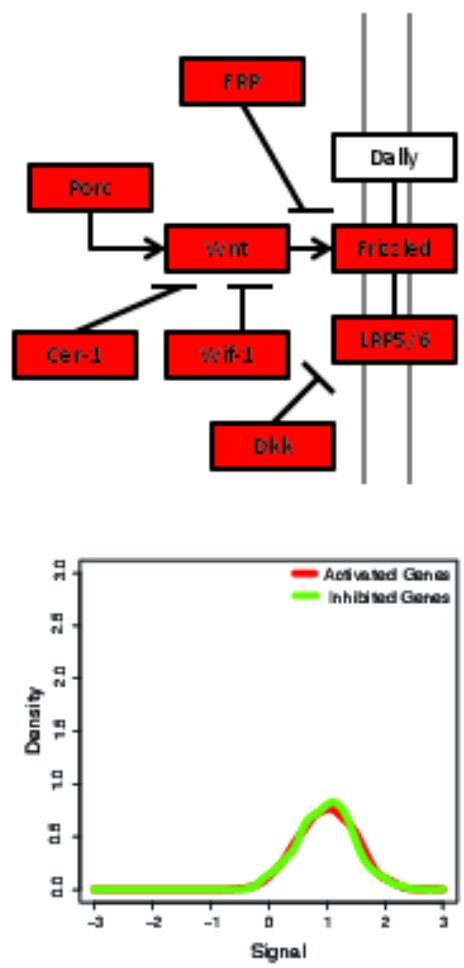


\section{Figure 2}

\section{BD-Func Recovers Known MSigDB Signatures}

This figure shows the output figures from BD-Func for the $5 \mathrm{MSigDB}$ signatures tested on their original datasets. A. Density plots for fold-change values for activated genes (colored red) and inhibited genes (colored green). These plots are used to illustrate BD-Func analysis on a single column of data (in this case, fold-change values between the positive and negative populations). B. Box-plots of activation versus inhibition test statistics for all relevant samples in each of the 5 MSigDB datasets. Note that each box-plot describes the distribution of test statistics for each group - it does not represent the expression of an individual gene or a metagene. In each of these five examples, the test statistic shows very clear differences among the different groups. If the median t-test statistic is greater than 2 , the box is colored red. If the median t-test statistic is less than -2 , the box is colored green.

\section{Figure 2: BD-Func Recovers Known MSigDB Signatures}

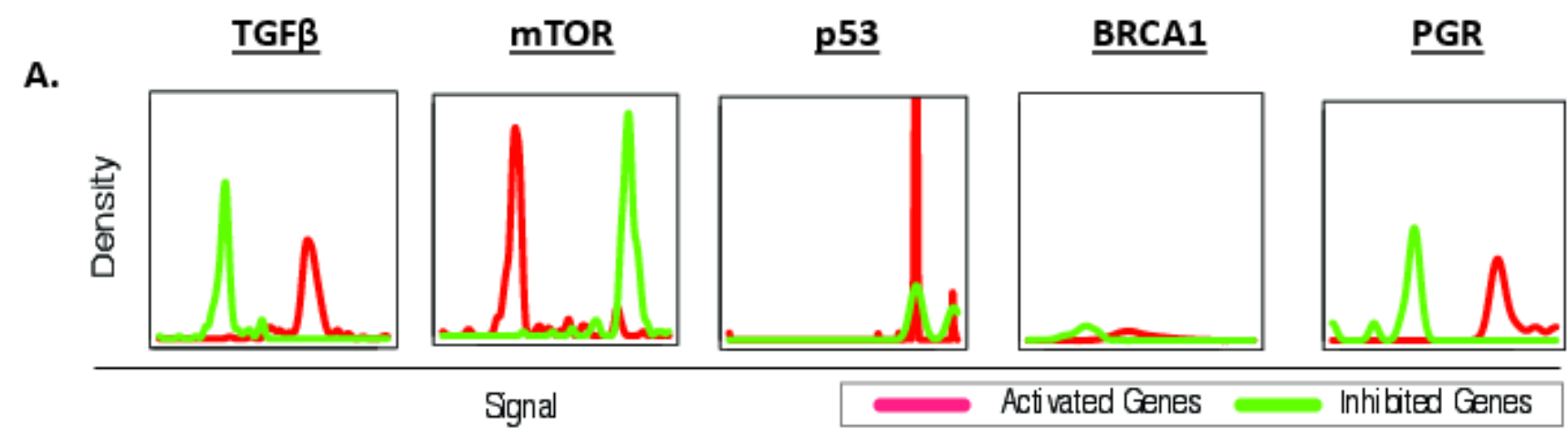

B.
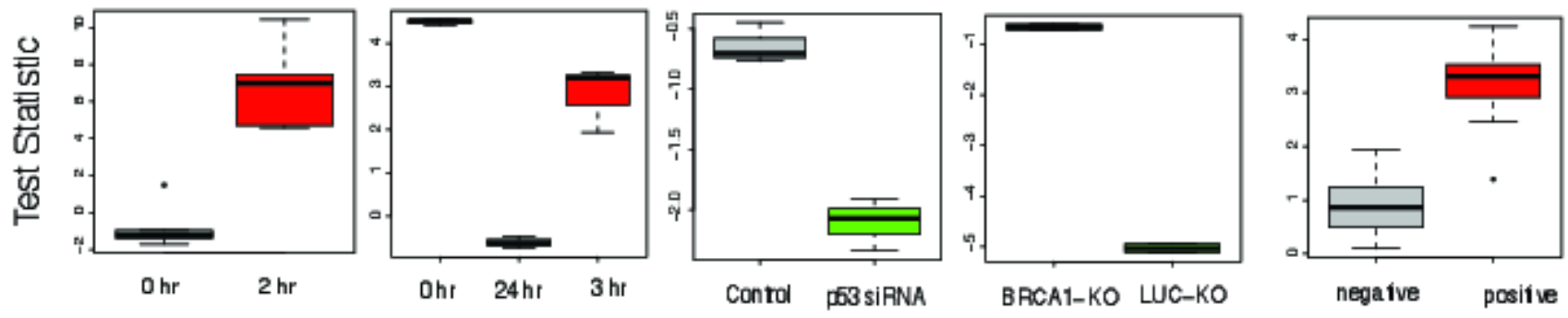


\section{Figure 3}

Custom Progesterone Signature Outperforms MSigDB Signature on Breast Cancer Patients

AUC (Area Under the Curve) values from the ROC plots for each patient cohort are displayed.

Although the MSigBD gene set shows extremely high accuracy for the original meningioma dataset, it shows essentially random predictive power for the 6 larger breast cancer datasets. On the other hand, a custom progesterone receptor signature defined using the expO dataset shows high accuracy for all 7 cohorts, and the high accuracy is maintained even in the largest cohort (TCGA).

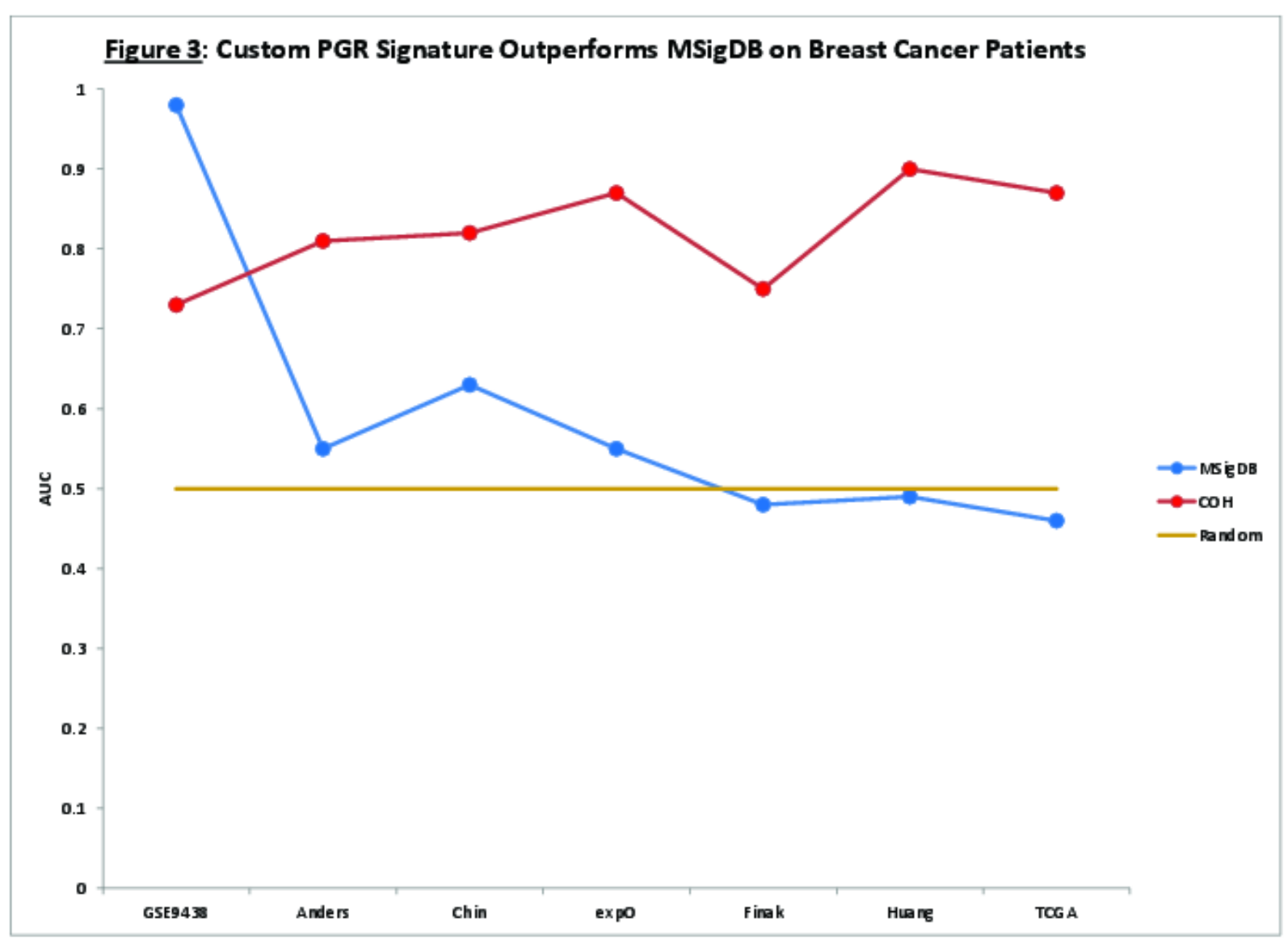




\section{Figure 4}

Novel Cell Line LBH589 Signature Can Accurately Detect Drug Activity In Vivo

A. BD-Func density plot for fold-change values for activated and inhibited genes for Exe + LBH589

vs. Exe alone tumors. At a population level, the Exe + LBH589 tumors show higher expression of activated genes whereas the Exe tumors show increased expression of inhibited genes $\left(p=2.0 \times 10^{-15}\right)$.

B. BD-Func box-plot for single-sample signature scores. EXE alone shows the greatest inhibition of LBH-related gene expression whereas EXE + LBH shows less inhibition of LBH-related gene expression, as

\section{Figure 4: Novel Cell Line LBH589 Signature Can Accurately Detect Drug Activity In Vivo}

A.

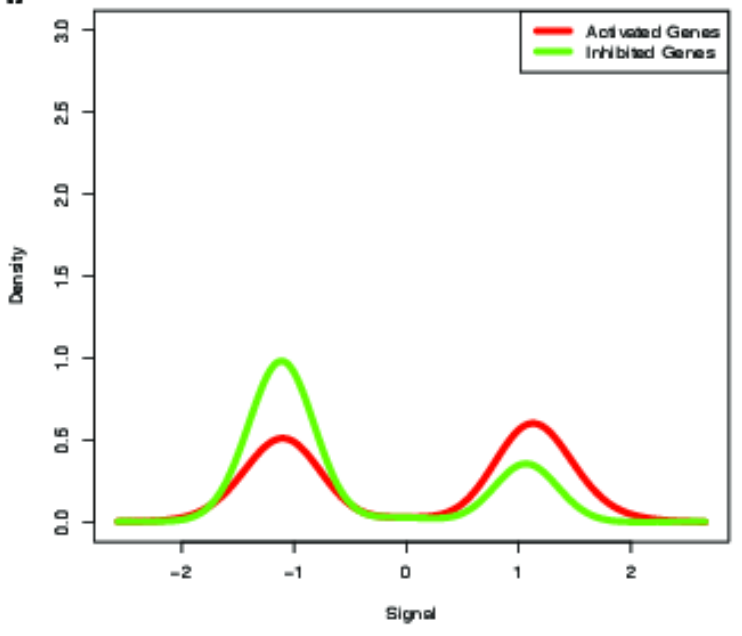

B.

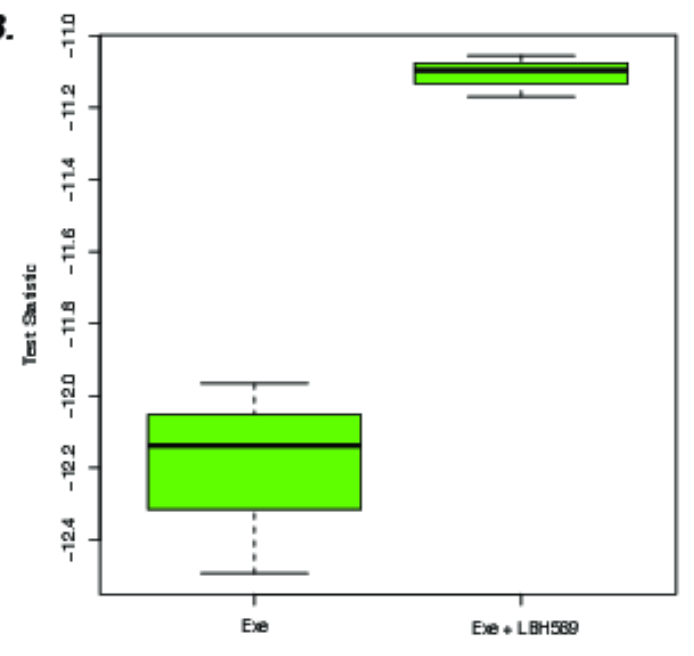




\section{Table $\mathbf{1}_{\text {(on next page) }}$ \\ Recoveryof Known Perturbations for Selected MSigDB Oncogenic Genes}

BD-Func "Fold-Change" corresponds to analysis of fold-change values calculated between the perturbed and unperturbed groups. BD-Func "Intensity" corresponds to calculation of activity vs. inhibition score for each sample in the dataset followed by a comparison in the distribution of test statistics for all of the samples. ${ }^{1}$ p5 53 signal changes with sign matching p53 expression (in this case P53_DN indicates genes down-regulated by knock-down of p53, not genes negatively related by p53) 
1 Table 1: Recovery of Known Perturbations for Selected MSigDB Oncogenic Genes

\begin{tabular}{|l|l|l|l|l|}
\hline & $\begin{array}{l}\text { BD-Func } \\
\text { (Fold-Change) }\end{array}$ & $\begin{array}{l}\text { BD-Func } \\
\text { (Intensity) }\end{array}$ & GSEA & IPA \\
\hline $\begin{array}{l}\text { TGF } \boldsymbol{\beta} \\
(\text { E-TABM-420) }\end{array}$ & Yes & Yes & $\begin{array}{l}\text { Yes (UP) } \\
\text { Yes (DN) }\end{array}$ & Yes \\
\hline $\begin{array}{l}\text { mTOR - N4 } \\
\text { (GSE5824) }\end{array}$ & Yes & Yes & $\begin{array}{l}\text { Yes (UP) } \\
\text { Yes (DN) }\end{array}$ & No \\
\hline $\begin{array}{l}\text { P53- V2 } \\
\text { (GSE1676 })\end{array}$ & Yes & Yes & $\begin{array}{l}\text { Yes (UP) } \\
\text { No (DN) }\end{array}$ & Yes \\
\hline $\begin{array}{l}\text { BRCA1 } \\
\text { (GSE4754) }\end{array}$ & Yes & Yes & $\begin{array}{l}\text { Yes (UP) } \\
\text { Yes (DN) }\end{array}$ & No \\
\hline $\begin{array}{l}\text { PGR } \\
\text { (GSE9438) }\end{array}$ & Yes & Yes & $\begin{array}{l}\text { No (UP) } \\
\text { No (DN) }\end{array}$ & No \\
\hline
\end{tabular}

2 


\section{Table 2 (on next page)}

\section{Prediction of TGF $\beta$ Activity in Novel Datasets}

${ }^{1}$ Qualitatively detected at 2 hours but not 4 hours, but activity is not significant with p-value $<0.05$ for either time-point 
Table 2: Prediction of TGF $\beta$ Activity in Novel Datasets

\begin{tabular}{|l|l|l|l|l|}
\hline & $\begin{array}{l}\text { BD-Func } \\
\text { (Fold-Change) }\end{array}$ & $\begin{array}{l}\text { BD-Func } \\
\text { (Intensity) }\end{array}$ & GSEA & IPA \\
\hline GSE1724 & Yes & Yes & $\begin{array}{l}\text { Yes (UP) } \\
\text { No (DN) }\end{array}$ & Yes \\
\hline GSE1805 & No & No $^{1}$ & $\begin{array}{l}\text { No (UP) } \\
\text { Yes (DN) }\end{array}$ & No \\
\hline GSE6653 & Yes & Yes & $\begin{array}{l}\text { Yes (UP) } \\
\text { Yes (DN) }\end{array}$ & Yes \\
\hline GSE17708 & Yes & Yes & $\begin{array}{l}\text { Yes (UP) } \\
\text { No (DN) }\end{array}$ & Yes \\
\hline
\end{tabular}




\section{Table 3 (on next page)}

Prediction of Progesterone Receptor Status inPatient Samples

In this table, BD-Func is used to analyze fold-change values between PR+ and PR-patients. MSigDB $=$ CLAUS_PGR_POSITIVE_MENINGIOMA signatures. COH $=$ novel PGR signature developed in this study. 
1 Table 3: Prediction of Progesterone Receptor Status in Patient Samples

2

\begin{tabular}{|l|l|l|l|l|}
\hline Cohort & $\begin{array}{l}\text { BD-Func } \\
\text { (MSigDB) }\end{array}$ & $\begin{array}{l}\text { BD-Func } \\
\text { (COH) }\end{array}$ & $\begin{array}{l}\text { GSEA } \\
\text { (MSigDB) }\end{array}$ & IPA \\
\hline $\begin{array}{l}\text { GSE9438 } \\
(\mathrm{N}=31)\end{array}$ & Yes & Yes & $\begin{array}{l}\text { No (UP) } \\
\text { No (DN) }\end{array}$ & No \\
\hline $\begin{array}{l}\text { Huang et al. 2003 } \\
(\mathrm{N}=88)\end{array}$ & No & Yes & $\begin{array}{l}\text { No (UP) } \\
\text { No (DN) }\end{array}$ & No \\
\hline $\begin{array}{l}\text { Chin et al. 2006 } \\
(\mathrm{N}=117)\end{array}$ & No & Yes & $\begin{array}{l}\text { No (UP) } \\
\text { No (DN) }\end{array}$ & No \\
\hline $\begin{array}{l}\text { Anders et al. 2008 } \\
(\mathrm{N}=73)\end{array}$ & No & Yes & $\begin{array}{l}\text { No (UP) } \\
\text { No (DN) }\end{array}$ & No \\
\hline $\begin{array}{l}\text { Finak et al. 2008 } \\
(\mathrm{N}=53)\end{array}$ & No & Yes & $\begin{array}{l}\text { No (UP) } \\
\text { No (DN) }\end{array}$ & No \\
\hline $\begin{array}{l}\text { expO } \\
(\mathrm{N}=256)\end{array}$ & No & Yes & $\begin{array}{l}\text { No (UP) } \\
\text { No (DN) }\end{array}$ & No \\
\hline $\begin{array}{l}\text { TCGA } \\
(\mathrm{N}=739)\end{array}$ & No & Yes & $\begin{array}{l}\text { No (UP) } \\
\text { No (DN) }\end{array}$ & No \\
\hline
\end{tabular}

3

4 